\title{
IQC analysis of constrained MPC of large-scale systems
}

DOI:

10.1016/B978-0-444-63965-3.50273-7

\section{Document Version}

Accepted author manuscript

Link to publication record in Manchester Research Explorer

\section{Citation for published version (APA):}

Petsagkourakis, P., Heath, W., \& Theodoropoulos, C. (2017). IQC analysis of constrained MPC of large-scale systems. In European Symposium on Computer-Aided Process Engineering https://doi.org/10.1016/B978-0-44463965-3.50273-7

\section{Published in:}

European Symposium on Computer-Aided Process Engineering

\section{Citing this paper}

Please note that where the full-text provided on Manchester Research Explorer is the Author Accepted Manuscript or Proof version this may differ from the final Published version. If citing, it is advised that you check and use the publisher's definitive version.

\section{General rights}

Copyright and moral rights for the publications made accessible in the Research Explorer are retained by the authors and/or other copyright owners and it is a condition of accessing publications that users recognise and abide by the legal requirements associated with these rights.

\section{Takedown policy}

If you believe that this document breaches copyright please refer to the University of Manchester's Takedown Procedures [http://man.ac.uk/04Y6Bo] or contact uml.scholarlycommunications@manchester.ac.uk providing relevant details, so we can investigate your claim.

\section{OPEN ACCESS}


Antonio Espuña, Moisès Graells and Luis Puigjaner (Editors), Proceedings of the $27^{\text {th }}$ European Symposium on Computer Aided Process Engineering - ESCAPE 27

October $1^{\text {st }}-5^{\text {th }}, 2017$, Barcelona, Spain (C) 2017 Elsevier B.V. All rights reserved.

\title{
IQC analysis of constrained MPC of large-scale systems
}

\author{
Panagiotis Petsagkourakis $^{\mathrm{a}}$, William Heath ${ }^{\mathrm{b}}$ and Constantinos Theodoropoulos ${ }^{\mathrm{a}^{*}}$ \\ ${ }^{a}$ School of Chemical Engineering and Analytical Science,The University of Manchester, \\ M13 9PL, UK \\ ${ }^{\mathrm{b}}$ School of Electrical and Electronic Engineering, University of Manchester, Manchester, \\ M13 9PL, UK \\ *K.Theodoropoulos@manchester.ac.uk
}

\begin{abstract}
Technological advances have led to the widespread use of more complex systems in both industrial and academic practice. Model predictive control (MPC) is a strong and widely used control methodology. MPC has been first applied to the chemical process industries and now it is also used in microelectronics. The advantage of MPC is that it can exploit a model of the process and, using the concept of moving horizons, it can find the optimum path for the manipulated variables. However, it is not straightforward or even computationally feasible to use MPC for distributed parameter systems consisting of systems of Partial Differential Equations (PDEs). This work exploits the dissipative nature that many engineering systems exhibit, for model order reduction (MOR) purposes. Dissipativity is expressed as separation of eigenvalues in the spectrum of the linearized system and therefore separation of modes into slow and fast ones. These types of problems contain uncertainties that affect the controller's stability. The stability and robustness of predictive control can be analyzed using the theory of integral quadratic constraints (IQCs). In this work we employ successive linearization off-line, constructing a model pool that consists of reduced size linear models. A dissipation inequality is then used to compute an upper bound for the input/output gain when MPC is employed, exploiting the IQCs to consider the uncertainties of the closed loop system. The effectiveness of the proposed method is demonstrated through two chemical engineering case studies.
\end{abstract}

Keywords: Robust stability, model predictive control, model reduction, integral quadratic constraints

\section{Introduction}

Model predictive control has found widespread use in the process industries (Mayne et al., 2006). However, it is still not trivial to guarantee robust stability under uncertainty (Heath and Li, 2010). Even the nominal stability of state feedback controllers needs state and terminal constraints Mayne et al. (2006). Heath and Li (2010) verify the stability of linear MPC under structured and unstructured uncertainties, extending the approach of (Heath et al., 2005) that provides nominal stability results for the output feedback MPC. The input/output analysis can guarantee global stability while a controller with terminal constraints can only provide local stability. Integral quadratic constraints (IQCs) (Megretski and Rantzer, 1997) have been used to perform robust analysis of dynamic and control 
systems. IQCs are used to describe the input/output behavior of nonlinear perturbations (uncertainties). Megretski and Rantzer (1997) introduced the IQC stability theorem in the frequency domain, that can be transformed to a linear matrix inequality (LMI) using the KYP lemma (Heath et al., 2005). Thus, the stability is verified if there exists a symmetric matrix $P=P^{T}$ satisfying LMI. Stability theorems have been introduced in the time domain using dissipation theory (Brogliato et al., 2007) and time domain IQCs (Pfifer and Seiler, 2014). In this case, the frequency domain IQCs can be expressed, using the Preserval's theorem. However, dissipation theory (Brogliato et al., 2007) requires the existense of a positive definite symmetric matrix. Model reduction plays an important role in MPC, as it is impossible to implement MPC in problems with a large number of variables (Theodoropoulos, 2011). These techniques can efficiently reduce the complexity of the system and effectively preserve its input-output properties. However, model reduction may include additional uncertainties. Hovland et al. (2008) exploited the work of (Løvaas et al., 2010) to provide a design methodology when model reduction (using balanced truncation) is applied in LTI models, although the implementation seems computationally intensive. The contribution of this work is to present a method to analyse the robustness of MPC using reduced piece-wise affine (PWA) systems in an input-output sense.

\section{Model Pool}

\subsection{Model reduction}

The physical plant is described by the following PDE-based system

$$
\frac{\partial x}{\partial t}=\Phi\left(x, \frac{\partial x}{\partial y}, \frac{\partial x^{2}}{\partial y^{2}}, u\right)
$$

where $\mathrm{x}$ and $\mathrm{u}$ are the states and inputs of the systems dynamics, respectively. An input/output black box is available for simulating the system given by Eq.(1). Thus, Eq.(1) is approximated using Eq.(2):

$$
x_{k+1}=F\left(x_{k}, u_{k}\right)
$$

Function $F(\cdot)$ in Eq.(2) can represent a black box simulator, which the user can only run for a given set of initial conditions, with given independent (control) variable profiles, for a set time. For most engineering systems there exists such a (potentially commercial) simulator. Let $P$ denote the maximal invariant subspace of the linearised system around a transient point, corresponding to the dominant modes and $Q$ its orthogonal complement. $\mathrm{P}$ and $\mathrm{Q}$ are the corresponding orthonormal projectors. It is important that there is only a small number of dominant modes to make the model reduction efficient. Let $Z \in \mathbb{R}^{N \times m}$ ( $N$ :number of variables and $m \ll N$ ) be the orthonormal basis of $P$ and now the projection $\mathrm{P}$ is computed as $\mathrm{P}=Z Z^{T}$. The orthonormal basis is used to project the state vector onto the dominant subspace. A new, reduced state vector is defined:

$$
\xi=Z^{T} x
$$

with $\xi \in \mathbb{R}^{m}$. This basis is also used for the computation of a reduced Jacobian. 


\subsection{Off-line successive linearisation of the reduced system}

The projection basis can be computed, with the availability of the simulator using only matrix (Jacobian)-vector products, which can be efficiently calculated with numerical directional perturbations (Bonis et al., 2014):

$$
F_{x} v=\frac{1}{2 \varepsilon}(f(x+\varepsilon v, u)-f(x-\varepsilon v, u))
$$

where $v$ the vector which needs to be multiplied with the Jacobian. The available dynamic simulator is used to perform these perturbations by perturbing the initial conditions and running the simulator for a certain reporting time. The states are used in the central finite difference scheme as in Eq.(2). Firstly, the product $F_{x} Z$ is computed by columns as in Eq.(4), performing $m$ perturbations, one for every column of $Z$. In the $j^{\text {th }}$ (of $m$ ) perturbations, the vector $v$ is actually the $j^{t h}$ column of $Z$. Subsequently, $F_{x} Z$ is pre-multiplied by $Z^{T}$ to form the desired reduced Jacobian, $A$. The above procedure is implemented in some collected trajectories creating the following state space representation.

$$
\begin{array}{r}
\xi_{k+1}=A_{i} \xi_{k}+B_{i} u \\
y_{k}=C_{i} x_{k}
\end{array}
$$

Eq.(5) represents the PWA system, created from a model pool consisting of reduced linear models. The controller selects which linear model will be used in the optimisation problem regarding the distance between the current reduced state and the collected points.

\section{Integral quadratic constraints (IQCs)}

This section briefly introduces the IQCs and more information can be found in Megretski and Rantzer (1997). IQCs provide a useful characterisation of a given operator on a Hilbert space defined in terms of self-adjoint operators. Let $\Pi$ be a bounded and selfadjoint operator, then $\Delta$ satisfies the IQC defined $\Pi$ if

$$
\left\langle\left[\begin{array}{c}
v \\
w
\end{array}\right], \Pi\left[\begin{array}{l}
v \\
w
\end{array}\right]\right\rangle=\int_{-\infty}^{\infty}\left[\begin{array}{c}
\widehat{v}(j \omega) \\
\widehat{w}(j \omega)
\end{array}\right]^{*} \Pi(j \omega)\left[\begin{array}{c}
\widehat{v}(j \omega) \\
\widehat{w}(j \omega)
\end{array}\right] d \omega \geq 0
$$

where $\widehat{v}$ and $\widehat{w}$ are the corresponding Fourier transformations, $\Pi$ is a multiplier that defines the IQC. Stability criteria have been introduced in the time-domain using dissipation theory and time domain IQCs (Pfifer and Seiler, 2014) exploiting the so-called hard IQCs. These are more generic than the soft constrains and hold at any finite moment $T$ (Megretski and Rantzer, 1997). This approach is based on the dissipation inequality (Brogliato et al., 2007) which requires the existence of a positive definite matrix. However, Pfifer and Seiler (2014) proposed a method to reduce the conservativeness for a particular class of IQC multipliers using (J-spectral factorisation). It is convenient to use time domain approaches as the burden of LTI can easily be surpassed. Similarly, the time domain hard IQC defined by $(\Psi, M)$ hold if the following inequality holds for all $r=\Psi\left[\begin{array}{c}v \\ w\end{array}\right]$ and $T$ :

$$
\sum_{k=0}^{T} r_{k}^{T} M r_{k} \geq 0
$$

The connection between frequency and time domain IQCs relies on the factorisation of the multiplier as $\Pi=\Psi^{*} M \Psi$. 


\section{Model predictive control}

We consider the MPC problem of a PWA system, assuming that only one linear model is used at every sampling time. The control law of the MPC with only input constraints (or at least $u=0$ is guaranteed feasible) can be seen as a static non-linearity $(\phi)$, which satisfies Eq.(8). It follows immediately from KKT conditions (Heath et al., 2005) that

$$
\phi^{T} H_{i} \phi-\phi^{T} s \leqslant 0
$$

where $\phi$ and $s$ are the input and output of the MPC respectively. Similarly to (Heath et al. 2005) every MPC law satisfies IQC with multiplier $\Pi=\left[\begin{array}{cc}0 & I \\ I & -2 H_{i}\end{array}\right]$.

\section{Dissipation inequality}

An uncertain PWA dynamical system $G_{i}$ can be described as in Figure 1. $\Delta$ represents the uncertainty and/or any other nonlinear part of the control scheme that satisfies an IQC. The induced $l_{2}$ gain from $d$ to $e$ is defined using the interconnection between $G$ and $\Delta$ as:

$$
\|G\|=\sup _{d \in l_{2}} \frac{\|e\|}{\|d\|}
$$

The uncertainty $\Delta$ is assumed to satisfy multiple time domain hard IQCs defined by the symmetric matrix $M$ and the filter $\Psi$. The robustness of the interconnected system and uncertaintiesnonlinearities are analysed using the extended system $G_{S}$ where the state space vector is $x_{s}:=\left[\begin{array}{l}x \\ \psi\end{array}\right]$. As in (Pfifer and Seiler, 2014) it is easy to prove that if there exists a symmetric positive definite matrix

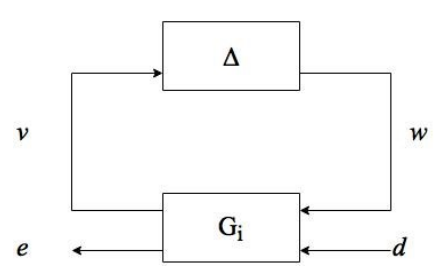

Figure 1: system's interconnection

$\Pi_{r}$ and $\lambda$ that satisfy the LMI in Eq.10, then the worst-case gain is less than $\gamma>0$, assuming that the open loop system $G_{s}$ is stable and all the uncertainties are bounded causal operators and/or satisfy IQCs. It should be mentioned that the following LMI includes only reduced linear models decreasing effectively the size of the constraints and the computational load.

$$
\begin{aligned}
L M I(\lambda, \gamma, \Pi) & :=\left[\begin{array}{ccc}
A_{s}^{i T} \Pi_{r} A_{s}^{i}-P & A_{s}^{i T} \Pi_{r} B_{s 1}^{i} & A_{s}^{i T} \Pi_{r} B_{s 2}^{i} \\
B_{s 1}^{i T} \Pi_{r} A_{s}^{i} & B_{s 1}^{i T} \Pi_{r} B_{s 1}^{i} & B_{s 1}^{i T} \Pi_{r} B_{s 2}^{i} \\
B_{s 2}^{i T} \Pi_{r} A_{s}^{i} & B_{s 2}^{i T} \Pi_{r} B_{s 1}^{i} & B_{s}^{i T} \Pi_{r} B_{s 2}^{i}-\gamma^{2} I
\end{array}\right]+ \\
+ & {\left[\begin{array}{c}
C_{s 2}^{i T} \\
D_{s 21}^{i T} \\
D_{s 22}^{i T}
\end{array}\right]\left[\begin{array}{c}
C_{s 2}^{i T} \\
D_{s 21}^{i T} \\
D_{s 22}^{i T}
\end{array}\right]^{i T}+\left[\begin{array}{c}
C_{s 2}^{i T} \\
D_{s 21}^{i T} \\
D_{s 22}^{i T}
\end{array}\right] M(\lambda)^{i}\left[\begin{array}{c}
C_{s 2}^{i T} \\
D_{s 21}^{i T} \\
D_{s 22}^{i T}
\end{array}\right]^{T} \leqslant 0 }
\end{aligned}
$$

\section{Applications}

\subsection{Tubular reactor}

To illustrate the features of the proposed analysis, we apply the framework to two different chemical engineering applications. The first one is a tubular reactor where an irreversible 


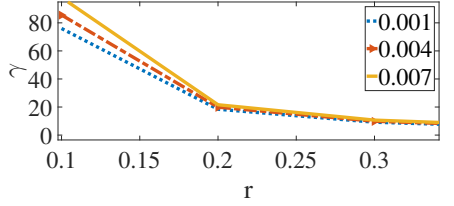

Figure 2: Solution for different model error limits

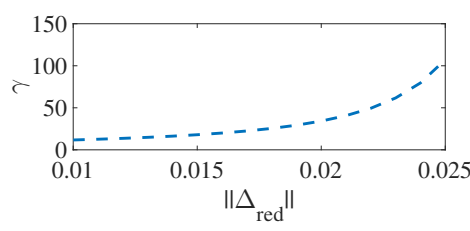

Figure 3: Stability against model error

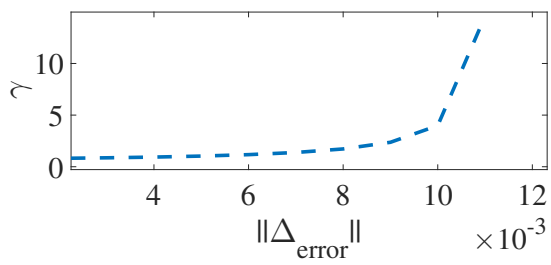

Figure 4: Stability against model error for evaporator

exothermic reaction takes place (Xie et al., 2015). The systems dynamics are described with the following dimensionless equations:

$$
\begin{aligned}
\frac{\partial c}{\partial t} & =\frac{1}{P e_{1}} \frac{\partial^{2} c}{\partial y^{2}}-\frac{\partial c}{\partial y}-D a c e^{\gamma_{c} T /(1+T)} \\
\frac{\partial T}{\partial t} & =\frac{1}{P e_{2}} \frac{\partial^{2} T}{\partial y^{2}}-\frac{\partial T}{\partial y}-B D a c e^{\gamma_{c} T /(1+T)}+b\left(T_{w}-T\right)
\end{aligned}
$$

with Neumann boundary conditions. Here $c$ and $T$ are the dimensionless concentration and temperature respectively, while $T_{w}$ is the temperature of the cooling zones representing the manipulated variables of the problem. In particular the cooling zones on the jacket of the reactor are separated in 8 different sectors. The parameters of the system are $P e_{1}=P e_{2}=7, D a=0.1, B=2, b=1$, and $\gamma_{c}=10$.

The model reduction is considered as norm bounded uncertainty that admits IQCs. The MPC also admits IQCs, with different static multipliers for each linear model. Stability analysis is carried out, where the objective is to find stability boundaries of the closed loop system. The degrees of freedom are the weight of the objective function in the optimization, $r$, and also the upper limit of the uncertainty of the model reduction. The analysis is crucial as it offers insight on how aggressive the controller can get, before the system becomes unstable, and also on how robust the controller is for high model errors. In figure 2 the worst case $\gamma$ is depicted against the parameter $r$ of the objective function for different upper limits of the model error. This analysis shows that the more aggressive the system is, the more difficult it becomes to guarantee the existence of finite gain $\gamma$ and that even for small upper bounds of the model reduction the system can become unstable as shown in Figure 2. For $r=0.5$, Figure 3 shows the effects of the model error. 


\subsection{Control of evaporator process}

The second application does not focus on showing how the methodology works with large scale systems, but rather how it works with a small PWA system with models in different operational regions (Kouramas and Dua, 2007). The system is linearised at two different operation points, therefore two different linear models are given. Particularly, the linear models are in the form of Eq. (1) with dynamics $A_{1}=\left[\begin{array}{ll}0.9054 & -0.0883 \\ -0.0093 & 0.9729\end{array}\right], A_{2}=$ $\left[\begin{array}{ll}0.9047-0.1008 \\ -0.0093 & 0.9726\end{array}\right]$ and $B_{1}=\left[\begin{array}{c}0.022764 .141 \times 10^{-} 5 \\ 0.0046-9.057 \times 10^{-}\end{array}\right], B_{2}=\left[\begin{array}{c}0.02593 .581 \times 10^{-} 5 \\ 0.0046-0.0007\end{array}\right]$, whilst it is assumed that we can measure all the states. For this example the stability is tested for a specific design parameter $r$. Thus, Figure 4 shows $\gamma$ against the norm-bound of the uncertainty of the model error depicting the expected trend. Nevertheless, in this case study, $\gamma$ is noticeably more sensitive as the error bound increases.

\section{Conclusion and Future work}

We have performed IQC-based stability analysis to reduced large-scale systems, accounting for the uncertainty that model reduction generates. This analysis can be performed to every reduced PWA system that is controlled by MPC. This also gives us the opportunity to avoid extensive data accumulation, as we can find the limits of our closed-loop system. One of the major issues of this analysis is that the model pool may be large making computations intractable. Thus, we plan to further reduce the number of linear models exploiting model reduction techniques. Furthermore, multiplier theory can be applied to reduce the conservativeness of the analysis.

\section{References}

Bonis, I., Xie, W., Theodoropoulos, C., 2014. Multiple model predictive control of dissipative PDE systems. IEEE Transactions on Control Systems Technology 22 (3), 1206-1214.

Brogliato, B., Lozano, R., Egeland, O., Maschke, B., 2007. Dissipative Systems Analysis and Control: Theory and Applications, 2nd Edition. Springer.

Heath, W., Li, G., 2010. Multipliers for model predictive control with structured input constraints. Automatica $46(3), 562-568$.

Heath, W., Li, G., Wills, A., Lennox, B., 2005. IQC analysis of linear constrained MPC. IEEE sponsored Colloquium on Predictive Control, Sheffield (July).

Hovland, S., Lovaas, C., Gravdahl, J. T., Goodwin, G. C., Dec 2008. Stability of model predictive control based on reduced-order models. In: 2008 47th IEEE Conference on Decision and Control. pp. 40674072.

Kouramas, K., Dua, V., 2007. Hybrid Parametric Model-Based Control. Wiley-VCH Verlag GmbH and Co. $\mathrm{KGaA}$.

Løvaas, C., Seron, M. M., Goodwin, G. C., 2010. Robust output-feedback MPC with integral action. IEEE Transactions on Automatic Control 55 (7), 1531-1543.

Mayne, D. Q., Raković, S. V., Findeisen, R., Allgöwer, F., 2006. Robust output feedback model predictive control of constrained linear systems. Automatica 42 (7), 1217-1222.

Megretski, A., Rantzer, A., 1997. System Analysis via Integral Quadratic Constraints Part II System Analysis via Integral Quadratic Constraints Part IIa : Abstract theory. Control 42 (September), 819-830.

Pfifer, H., Seiler, P., 2014. Robustness analysis of linear parameter varying systems using integral quadratic constraints. International Journal of Robust and Nonlinear Control 25 (October 2014), 2843-2864.

Theodoropoulos, C., 2011. Optimisation and linear control of large scale nonlinear systems: A review and a suite of model reduction-based techniques. In: Lecture Notes in Computational Science and Engineering Vol 75. Springer, pp. 37-61.

Xie, W., Bonis, I., Theodoropoulos, C., 2015. Data-driven model reduction-based nonlinear MPC for largescale distributed parameter systems. Journal of Process Control 35, 50-58. 